\title{
Conceptualization of category-oriented likelihood ratio: a useful tool for clinical diagnostic reasoning
}

\author{
Hamideh Moosapour ${ }^{1 \dagger}$, Mohsin Raza ${ }^{2 \dagger}$, Mehdi Rambod ${ }^{3 \dagger}$ and Akbar Soltani ${ }^{*}$
}

\begin{abstract}
Background: In the diagnostic reasoning process medical students and novice physicians need to be made aware of the diagnostic values of the clinical findings (including history, signs, and symptoms) to make an appropriate diagnostic decision. Diagnostic reasoning has been understood in light of two paradigms on clinical reasoning: problem solving and decision making. They advocate the reasoning strategies used by expert physicians and the statistical models of reasoning, respectively. Evidence-based medicine (EBM) applies decision theory to the clinical diagnosis, which can be a challenging topic in medical education.

This theoretical article tries to compare evidence-based diagnosis with expert-based strategies in clinical diagnosis and also defines a novel concept of category-oriented likelihood ratio $(L R)$ to propose a new model combining both aforementioned methods.

Discussion: Evidence-based medicine advocates the use of quantitative evidence to estimate the probability of diseases more accurately and objectively; however, the published evidence for a given diagnosis cannot practically be utilized in primary care, especially if the patient is complaining of a nonspecific problem such as abdominal pain that could have a long list of differential diagnoses. In this case, expert physicians examine the key clinical findings that could differentiate between broader categories of diseases such as organic and non-organic disease categories to shorten the list of differential diagnoses. To approach nonspecific problems, not only do the experts revise the probability estimate of specific diseases, but also they revise the probability estimate of the categories of diseases by using the available clinical findings.
\end{abstract}

Summary: To make this approach analytical and objective, we need to know how much more likely it is for a key clinical finding to be present in patients with one of the diseases of a specific category versus those with a disease not included in that category. In this paper, we call this value category-oriented $L R$.

\section{Background}

Diagnostic reasoning is one of the most difficult clinical skills particularly in the primary care setting. History taking and physical examination form the foundations of diagnostic reasoning [1]. Taking an adequate history and physical examination that suggest possible differential diagnoses are very important, so that without an adequate history and physical examination, all the

\footnotetext{
* Correspondence: soltania@tums.ac.ir

+ Contributed equally

${ }^{1}$ Evidence-Based Medicine \& Critical Thinking Working Team, Endocrinology and Metabolism Research Institute, Tehran University of Medical Sciences,

Tehran, IR Iran

Full list of author information is available at the end of the article
}

subsequent diagnostic investigations might be misleading [2]. Despite its importance, significant weaknesses in the diagnostic skills among medical students, residents, and practicing physicians have been consistently reported $[3,4]$. Studies have shown that the most common error in the diagnostic reasoning of medical students and novice physicians is the use of nondiscriminatory clinical findings (i.e. signs and symptoms) to support a given clinical diagnosis $[5,6]$.

Medical students are usually taught to take a thorough history and perform detailed physical examination for all patients, which is quite different from the strategies that expert physicians use to make diagnosis [7]. Expert physicians use some key clinical findings to make a

\section{Biomed Central}


diagnosis or to decrease the number of differential diagnoses in a relatively short period of time. An expert physician uses such discriminatory signs and symptoms subjectively, while students, novice physicians and clinical educators need to be objectively aware of the diagnostic value of these discriminatory signs and symptoms in order to differentiate different diseases. Although the physicians' intuitive clinical experience is usually the main source of learning about the diagnostic value of clinical findings, such unsystematic experience may not be as accurate as the information obtained from highquality diagnostic studies [8].

Diagnostic reasoning has been understood in the light of two paradigms for psychological research on clinical reasoning: problem solving and decision making. The former describes and advocates the reasoning strategies used by expert physicians to improve the instruction of medical students; the latter, on the other hand, emphasizes the statistical models of reasoning. Evidence-based medicine (EBM) applies decision theory to the clinical diagnosis [9], which can be a challenging topic in medical education. This article aims at introducing the new concept for revising the probability estimates of a whole category of diseases (instead of one specific disease) which we call "categoryoriented likelihood ratio" (LR) hereafter in evidence based clinical diagnosis. Also the potential application of category-oriented LRs, as a novel strategy for evidence-based clinical diagnosis, is proposed and discussed in this article.

\section{Discussion}

\section{Evidence-Based clinical diagnosis and its limitations}

In the following sections, we describe the use of LRs in revising the probability of diseases. In order to make a better understanding of the concept of disease-oriented LR and category-oriented LR in diagnostic reasoning, we first outline two clinical scenarios below. Then we will use these two clinical cases in the rest of this publication.

\section{Clinical scenarios}

Case 1: A 24-year-old healthy woman presents to a primary care physician complaining of an increased urinary frequency and burning pain during urination. She has had two episodes of prior urinary tract infections (UTI), and this episode seems "just like the previous one". She is sexually active with one partner and uses condoms with spermicidal jelly. Her physician considers UTI as the most probable diagnosis for the patient's specific complaints. The physician asks for and she denies having fever, back pain, nausea, vomiting, vaginal discharge, and hematuria. Here, following questions can be considered for clinical diagnosis:

- What is the diagnostic value of the presence or absence of the clinical findings such as dysuria, frequency or self-diagnosis in the diagnosis of UTI?
- How can a clinical educator objectively teach the students about revising the probability estimate of a differential diagnosis?

Case 2: A primary care physician is evaluating a 38year-old woman who complains of generalized abdominal pain from 3 months ago. The physician knows the long list of differential diagnoses such as disorders of stomach function and/or gastritis, infectious diarrhea, appendicitis, acute cholecystitis, diaphragmatic hernia, ulcerative colitis, malignant neoplasm of kidney, etc. which can be potentially the cause of the patient's nonspecific problem. In this scenario, following questions can be worthy of consideration:

- How should the primary care physician approach this non-specific problem in his patient?

- What are the important questions in history and physical examination in approaching this patient with a non-specific problem?

- Which important findings should the physician seek for?

- How would the physician advance from such a non-specific presentation to the potential differential diagnoses and then, to the final diagnosis?

- How could a clinical educator objectively teach diagnostic reasoning to the medical students to approach similar cases?

\section{Use of LRs to revise the probability estimate of a given disease}

Medical students and novice physicians may not be clear enough about the exact diagnostic values of various clinical findings for a given diagnosis. This might partly have its origin in the utilization of traditional medical literature by medical students as the sources of knowledge rather than evidence-based literature [10]. In traditional medical literature, the signs and symptoms of a disease are generally listed without their predictive power in making a diagnosis [8]. For example, according to the traditional medical, frequency and dysuria support the diagnosis of urinary tract infection (UTI); the literature, however, does not yield any information about the relative diagnostic value of these clinical findings for the diagnosis of UTI objectively; nor does the traditional medical literature provide any quantitative information to estimate the probability of uncomplicated UTI in the presence or absence of such clinical findings (i.e. dysuria and frequency).

Evidence-based diagnosis attempts to improve the clinical diagnosis by applying research-derived evidence to the diagnostic decision-making process. The probabilistic reasoning in evidence-based diagnosis conveys the presence of 
uncertainty in every clinical decision. Using probabilistic reasoning, a diagnosis cannot (and doesn't need to be) completely ruled in/out. Instead, the probability estimate needs to be revised accurately for a disease to decrease the uncertainty and to exactly determine a physician's position regarding a predefined diagnostic threshold [11]. From this point of view, diagnostic reasoning is the process of revising the probability of having a disease by using new clinical and para-clinical data. Bayes' theorem is the standard approach to revise probabilities [9]. By applying Bayes' theorem to the new data obtained from clinical or para-clinical tests, the previous estimation of a disease probability could be revised [12-14]. For instance, the probability of UTI in case 1 would be revised depending on the presence or absence of hematuria using an objective and userfriendly diagnostic tool, known as likelihood ratio (LR).

Likelihood ratios indicate how much more likely it is for a test or a clinical finding to give a positive or negative result in a patient versus an individual free of the disease. Likelihood ratios are calculated from diagnostic studies as follow $[15,16]$ :

$$
\text { The likelihood ratio for a positive test }[\mathrm{LR}(+)]=\frac{\text { sensitivity }}{1-\text { specificity }}
$$$$
\text { The likelihood ratio for a negative test }[\operatorname{LR}(-)]=\frac{1-\text { sensitivity }}{\text { specificity }}
$$

The more distant the LR from 1, the more valuable it is for making a diagnosis. As a rule of thumb, $\mathrm{LR}(+)>$ 10 or $\operatorname{LR}(-)<0.1$ generate large and often conclusive changes from pre- to post-test probability and the likelihood ratios of 5 to 10 and 0.1 to 0.2 generate moderate shifts in pre- to post-test probability $[15,16]$.

Table 1 shows the diagnostic accuracy of clinical symptoms and signs in the prediction of uncomplicated UTI [17]. This table shows that self-diagnosis has the greatest diagnostic value, which is twice the diagnostic value of hematuria. By applying simple formulae or a nomogram, the LRs can revise the pre-test probability estimate into a post-test probability (Table 2), while the pre-test probability is either the known prevalence of a disease or a physician's subjective impression of the probability of the disease in a given patient $[15,16]$. For example, according to table 1 , the post-test probability of uncomplicated UTI in Case 1 is equal to $81 \%$ (Table 2).

It should be mentioned that several LRs can be used together sequentially to calculate a single estimate of post-test probability, if the different clinical findings are independent. However, if the clinical findings are not independent, it is wiser to use the one with the better LR. For example, from two dependent clinical findings in Case 1, i.e. vaginal discharge and vaginal irritation, we only use LR (-) for vaginal discharge because it has a better discriminatory value and more reliability
Table 1 Diagnostic accuracy of symptoms and signs in predicting urinary tract infection as measured by positive and negative likelihood ratio (LR)*

\begin{tabular}{lcc}
\hline Symptom/Sign & Positive LR & Negative LR \\
\hline Dysuria & 1.5 & 0.5 \\
Frequency & 1.8 & 0.6 \\
Hematuria & 2 & 0.9 \\
Fever & 1.6 & 0.9 \\
Flank Pain & 1.1 & 0.9 \\
Lower Abdominal Pain & 1.1 & 0.9 \\
Vaginal Discharge & 0.3 & 3.1 \\
Vaginal Irritation & 0.2 & 2.7 \\
Back Pain & 1.6 & 0.8 \\
Self-diagnosis & 4 & 0.1 \\
Vaginal Discharge on Physical Examination & 0.7 & 1.1 \\
Costovertebral Angle Tenderness & 1.7 & 0.9 \\
Dipstick Urinalysis\# & 4.2 & 0.3 \\
\hline
\end{tabular}

* Values are derived from the study by Bent et al. [17]

\# A positive result was defined as leukocyte esterase positive or nitrite positive, a negative result was defined as both negative.

compared to the vaginal irritation. In situations similar to Case 1 that present with specific problems, the physicians can first formulate the differential diagnoses such as UTI and vaginitis. Then LRs can inform the physician about the value of different clinical findings to estimate the probability of each differential diagnosis.

In spite of the above-mentioned UTI case, most patients referring to primary care physicians have presenting complaints of nonspecific problems such as abdominal pain (as in Case 2) that have potentially a long list of differential diagnoses. In these circumstances, physicians may not formulate an earlier list of specific diseases for differential diagnosis. Also, disease-oriented LRs cannot help formulate objectively the differential diagnosis list either. Therefore, the LR-based diagnostic approach is not in line with the expert physicians' usual approach to nonspecific clinical problems [18].

\section{Table 2 Calculation of post-test probability using} likelihood ratios

$$
\begin{aligned}
& \text { 1. } \quad \text { Odds }=\frac{\text { probability }}{1-\text { probability }} \\
& \text { 2. Post-test odds }=\text { pre-test odds } \times \text { likelihood ratios } \\
& \text { For example, according to table 1, post-test probability of } \\
& \text { uncomplicated UTI in case1 can be calculated as: } \\
& \text { Pre-test odds }=\frac{0.12}{(1-0.12)}=0.13 \\
& \text { Post-test odds }=0.13 \times 1.5 \times 1.8 \times 3.1 \times 4=4.35 \\
& \text { Post-test probability }=\frac{4.35}{(1+4.35)}=81 \%
\end{aligned}
$$


An evidence-based diagnostic approach has some theoretical and practical limitations. LRs in evidence-based literature are disease-oriented and can only be used to diagnose a given disease, i.e. to revise the probability estimate of a given differential diagnosis. To formulate and to further decrease the number of differential diagnoses in the case of nonspecific clinical problems, another objective tool is needed. The following sections introduce the category-oriented $L R$, as the new objective tool.

\section{Expert-based clinical diagnosis and their drawbacks}

\section{Conceptualization of revising category probability estimates}

Moving from a clinical presentation towards a final diagnosis is not a predetermined procedure. Psychological research on problem-solving paradigm shows that expert physicians use several alternative strategies in clinical diagnosis depending on their clinical experience and familiarity with the problem that is presented to them [19]. Three different diagnostic reasoning strategies available to learners include [19]:

A. pattern recognition

B. hypothetico-deductive

C. scheme-inductive problem solving

The first strategy, pattern recognition, is an intuitive, experience-based process where clinical decisions are made rapidly and almost autonomically by perceiving that clinical findings from a new patient resemble a previously learned illness script [9]. In the second strategy, hypothetico-deductive problem solving, when patients present with a medical problem, physicians begin by generating a list of specific medical conditions that could logically explain the patient's problems namely differential diagnoses. Most physicians keep 3-5 differential diagnoses in their mind at one time which include: more common, more serious if undiagnosed and/or untreated, and more responsive to treatment [20].

In the case of specific problems, early formulation of differential diagnoses is straightforward. However, when nonspecific problems such as abdominal pain, fatigue, anorexia, fever, and weakness are encountered as presenting complaints, formulating a short differential diagnosis list would be complicated and cumbersome. In these situations, it may be useful to first think about the problem in terms of how it might be classified or categorized by etiology or other associated features such as anatomy, pathophysiology, body system, or more generic disease categories such as infectious, metabolic, neoplastic or psychiatric diseases and so on. At this step, looking for key clinical findings that can differentiate between the broader categories of diseases can help the physician decrease the number of differential diagnoses. For example, in approaching to fever, the presence of bone pain and weight loss suggests category of malignancy, the presence of rash and arthritis suggests category of inflammatory diseases, and the history of exposure to febrile patients or recent traveling suggests category of infectious diseases [19].

Teaching the third strategy mentioned above to medical students should be guided by a scheme. Various schemes have been considered to reflect an organized knowledge structure for the purpose of learning as well as providing a structure for diagnostic reasoning. By following such schemes, the physician actually looks for key clinical findings that will help him distinguish between the categories of conditions at the branching points of the scheme. The presence or absence of these clinical findings leads to the adoption of one category and the exclusion of the others. After several branching points, when the number of diagnostic options has been considerably reduced, the deductive reasoning or pattern recognition may be utilized [19]. Throughout this diagnostic reasoning strategy, especially in approaching the nonspecific problems, not only do the physicians revise the probability estimate of single diseases, but also they mostly revise the probability estimate of the categories of diseases. For example, if a patient is complaining of fatigue, lack of fever is a key clinical finding that decreases the probability of infectious disease category or lack of dyspnea on exertion is a key clinical finding that decreases the probability of cardiac disease category.

In Case 2, an expert primary care physician is evaluating a 38-year-old woman with abdominal discomfort. The physician knows that the abdominal pain could be due to a disease from a long list of differential diagnoses including disorders of stomach function and/or gastritis, infectious diarrhea, appendicitis, acute cholecystitis, diaphragmatic hernia, ulcerative colitis, malignant neoplasm of kidney, etc [21]. The physician does not and cannot consider each of the diseases and use key clinical findings to distinguish them separately. However, before thinking about them separately and considering each of them as a differential diagnosis, a primary care physician would discern that the patient has acute or non-acute abdominal complaints and so he asks about the onset of problems (Figure 1). The patient says that her problem began almost 3-months ago. Considering that a nonacute abdominal complaint could be due to an organic or a non-organic disease, the physician considers evaluating the probability of the category of organic diseases in the patient. So he then asks for some clinical findings that have potential discriminatory power between the organic versus non-organic categories [21].

At this point, the physician asks about the history of blood in stool, pain affecting sleep, pain relief after defecation, the specific characteristic of the pain, and weight 


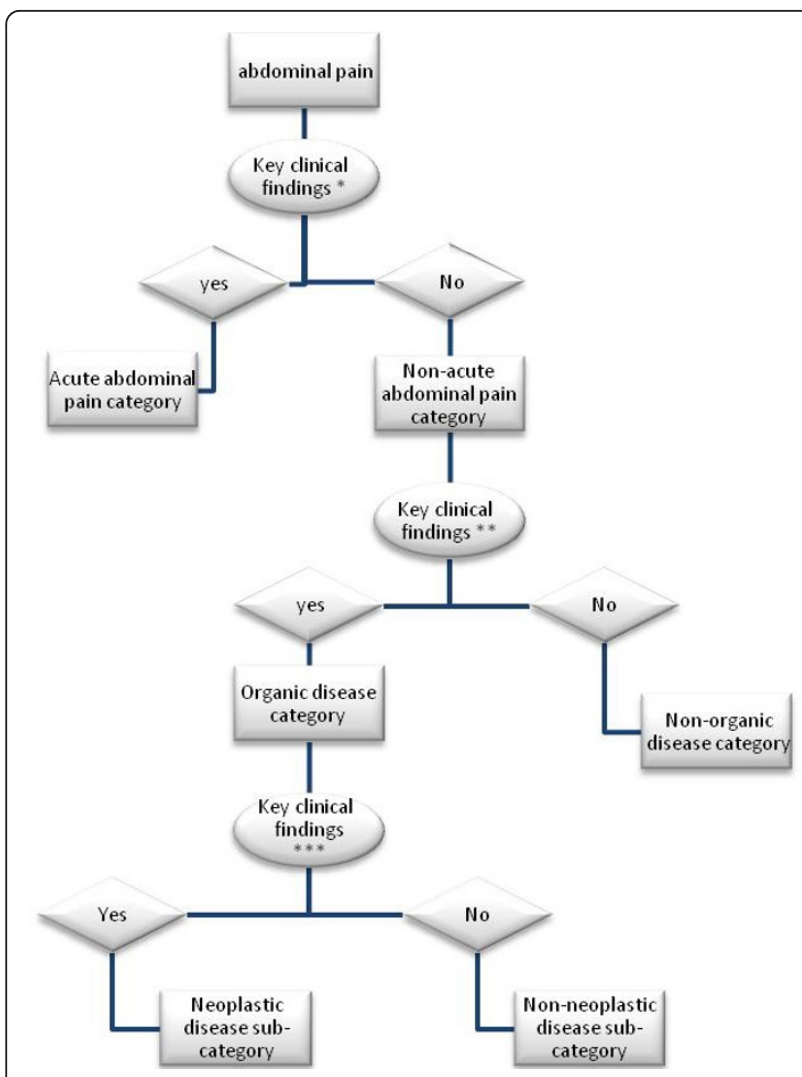

Figure 1 An example for the scheme of an expert physician to approach abdominal pain! 'This is an example for the experts' scheme of abdominal pain and not derived from a qualitative relevant study. ${ }^{*}$ Onset of symptom. ${ }^{*}$ male gender, age $>60$ years, history of blood in stool, pain affecting sleep, no pain relief after defecation, no specific character of pain, weight loss $>1 \mathrm{~kg}$ in 4 weeks. ${ }^{* *}$ White blood cell count $>10,000 \mathrm{~mm}^{-3}$, erythrocyte sedimentation rate $>20 \mathrm{~mm}$ per hour, low hemoglobin level.

loss $>1 \mathrm{~kg}$ in 4 weeks. The patient mentions her pain relief after defecation and the fact that the pain does not affect her sleep. There is neither specific character to pain (such as description of the pain as one or more of the following: burning, cutting, terrible, feeling of pressure, dull, boring), nor significant weight loss nor history of blood in stool. At this point, non-organic category will be more probable and organic category less probable in the physician's mind.

The physician can again revise the differential diagnoses to evaluate the probability of a fatal sub-category of the organic category, i.e. neoplasm category. Hence, according to the key laboratory findings ordered for this purpose [21], the physician orders CBC and ESR for the patient. Normal WBC, hemoglobin and ESR convince the physician to treat the patient symptomatically, follow her up and hold further evaluation or refer to a specialist.

Therefore, by asking only few key questions and knowing the value of the discriminatory powers of such clinical findings, the physician can revise the probability estimates of the categories that include all pertaining diseases. In this step, the category of the disease is investigated.

At a critical point, these categorizations are not always mutually exclusive and most of these clinical findings may not be totally discriminative. Hence, the presence or absence of a clinical finding at a particular branching point can only increase or decrease the previous probability of those categories and does not rule in/out the diseases.

An important query which arises here is how to quantify the change in the probability of a category of diseases given a particular clinical sign or symptom. For example, in Case 2, the history of pain that does not affect sleep cannot totally rule in non-organic category or rule out organic category. However, like every other clinical finding, the pain that does not affect sleep can potentially decrease or increase the probability of a certain category. This example elucidates that expert physicians can subjectively revise the probability estimate of a disease category based on their past clinical experience, in the same fashion as they revise probabilities estimate of a disease. Medical students however may not easily understand and follow this approach. Therefore, clinical educators should explicitly teach the objective and quantified diagnostic value of these clinical findings to the medical students.

On the other hand, scheme-inductive problem solving and scheme trees serve as pathways for teaching, learning and clinical problem solving in some medical curricula [22]. However, these schemes and key clinical findings are mostly based on experts' personal experience and beliefs and may not be necessarily based on valid evidence. Expert physicians reach to the key clinical findings via trials and errors throughout several years of their clinical practice that may involve some degrees of cognitive biases [23,24]. Research on diseaseoriented key clinical findings to revise the probability estimate of a given disease has shown that such clinical findings may be far less useful while considering their positive or negative likelihood ratios. For example, rebound tenderness, which is considered by many physicians as the diagnostic hallmark of acute appendicitis, has a positive LR of 1.9 [25]. This implies that a physician has to be more objective and vigilant while considering category-oriented key clinical findings as well as disease-oriented ones.

\section{Conceptualization of Category-Oriented Likelihood Ratio}

A key question in dealing with patients presenting with non-specific complaints is whether a characteristic of the complaint or another sign/symptom can differentiate between two major categories of diseases. In the above 
scenario, the physician wants to know whether a pain that awakens the patient can discriminate between organic causes vs. non-organic etiologies. And if so, what the post-test probability of a certain category would be? While EBM emphasizes that key clinical findings for the diagnosis of a given disease are nothing but signs or symptoms with significant likelihood ratios (disease-oriented LRs) [26], the aforementioned questions will be answered by novel concept of category-oriented $L R$ as defined in this article.

Let's return to Case 2 and solve it objectively by category-oriented LRs. Regarding the prevalence, the pre-test probabilities of organic and non-organic categories are $14 \%$ and $86 \%$ respectively (Table 3 ) [27]. In order to revise the probability estimate of the organic category in approaching patients with non-acute abdominal pain, we have calculated category-oriented LRs for the most discriminatory clinical findings by using sensitivities and specificities from a study published by Muris et al. (Table 4) [27]. According to age and sex and after negative answers to 5 key discriminatory questions in the patient's history, the probability of organic category decreased to $4 \%$ and the probability of non-organic category increased to $96 \%$ (Table 5, Figure 2). Normal values of the 3 key discriminatory para-clinical findings again decreased the probability of organic to $3 \%$ and increased that of non-organic to $97 \%$. Finally, the probability of neoplasm sub-category decreased using category-oriented LRs from $4 \%$ to almost $0.8 \%$ (Table 5).

This step generally deals with the 'category' of the disease rather than with the disease itself. To perform this task efficiently, the category-oriented LRs are inevitably needed. Therefore, by having category-oriented LRs and asking only few appropriate questions, the physician can objectively reduce the probability of a large number of differential diagnoses and limit the list of differential diagnosis.

\section{Summary}

In this theoretical article, we attempted to compare and contrast evidence-based diagnosis and expert-based strategies in clinical diagnosis and proposed a new model combining both aforementioned methods. We also proposed the concept of category-oriented $L R$ and suggested that the values of category-oriented LRs need to be calculated and tested in future studies. We believe that, not only can EBM properly use disease-oriented LRs to revise the probability estimate of given diseases, but it could also be helpful to revise the probability estimate of the category of diseases by using category-oriented LRs.

At this time, there is an increasing body of critically reviewed literature that emphasizes the diagnostic utility of specific physical findings [3,28]. Available systematic
Table 3 Final diagnoses in the patients with non-acute abdominal pain*

\begin{tabular}{|c|c|}
\hline Final diagnosis & $\%$ of patients \\
\hline \multicolumn{2}{|l|}{ Non-organic } \\
\hline Abdominal symptoms (no diagnosis) & 63.1 \\
\hline Disorders of stomach function/gastritis & 7.6 \\
\hline irritable bowel syndrome & 14.8 \\
\hline \multicolumn{2}{|l|}{ Organic } \\
\hline infectious diarrhea, dysentery & 0.4 \\
\hline other presumed infections & 1.1 \\
\hline malignant neoplasm stomach & 0.2 \\
\hline Malignant neoplasm colon, rectum & 0.4 \\
\hline malignant neoplasm pancreas & 0.2 \\
\hline malignant neoplasm other and unspecified sites & 0.2 \\
\hline benign neoplasms (digestive tract) & 0.9 \\
\hline Disease of oesophagus & 0.4 \\
\hline Duodenal ulcer & 1.7 \\
\hline other peptic ulcers & 1.0 \\
\hline Appendicitis & 0.1 \\
\hline inguinal hernia & 0.1 \\
\hline hiatus (diaphragm) hernia & 0.3 \\
\hline other abdominal hernia & 0.1 \\
\hline diverticular diseases of intestines & 1.4 \\
\hline chronic enteritis/ulcerative colitis & 1.3 \\
\hline anal fissure/perianal abscess & 0.4 \\
\hline cholecystitis/cholelithiasis & 0.3 \\
\hline other disease digestive system & 0.1 \\
\hline Haemorrhoids & 0.6 \\
\hline malignant neoplasm trachea/bronchus/lung & 0.2 \\
\hline pyelonephritis/pyelitis, acute & 0.1 \\
\hline cystitis/other urinary infection & 0.2 \\
\hline malignant neoplasm kidney & 0.1 \\
\hline urinary calculus & 0.4 \\
\hline other disease of urinary system & 0.2 \\
\hline malignant neoplasm cervix & 0.1 \\
\hline other malignant neoplasm (female genital system) & 0.1 \\
\hline fibroid/myoma (uterus/cervix) & 0.9 \\
\hline other diseases female genital tract & 0.6 \\
\hline
\end{tabular}

* Values are derived from the study by Muris et al. [27]

reviews present the accuracy of symptoms and signs in the diagnosis of different diseases in terms of sensitivity, specificity and LRs [29]. These systematic reviews also propose various diagnostic algorithms and/or scoring systems as diagnostic prediction rules that are based on the best available evidence [29]. Such tools are diseaseoriented, too. Future studies are needed to calculate exact values for the category-oriented LRs of different clinical presentations in determining the various categories of diseases. We suggest the systematic and scientific empirical testing of experts' diagnostic schemes of different clinical presentations by disease-oriented and category-oriented LRs for objectivity and accuracy 
Table 4 Category-oriented likelihood ratios (LR) of signs and symptoms and laboratory results for organic diseases in patients with non-acute abdominal pain*

\begin{tabular}{|c|c|c|}
\hline Patient Characteristics & Category-oriented LR(+) & Category-oriented LR(-) \\
\hline Male sex & 1.41 & 0.78 \\
\hline Age $>30$ years & 1.12 & 0.64 \\
\hline Age $>60$ years & 1.47 & 0.9 \\
\hline $30<$ Age $<60$ years & 1.08 & 0.88 \\
\hline \multicolumn{3}{|l|}{ Symptoms } \\
\hline History of blood in stool & 1.5 & 0.89 \\
\hline Pain affecting sleep & 1.3 & 0.77 \\
\hline No pain relief after defecation & 1.1 & 0.72 \\
\hline No specific character to pain ${ }^{\#}$ & 1.5 & 0.93 \\
\hline Weight loss $>1 \mathrm{~kg}$ in 4 weeks & 1.29 & 0.89 \\
\hline \multicolumn{3}{|l|}{ Laboratory tests } \\
\hline White blood cell count $>10000 \mathrm{~mm}^{-3}$ & 2.28 & 0.9 \\
\hline Erythrocyte sedimentation rate $>20 \mathrm{~mm}$ hour' & 2 & 0.92 \\
\hline Low hemoglobin level & 1.78 & 0.87 \\
\hline
\end{tabular}

* Category-oriented LRs calculated using values of sensitivity and specificity derived from the study by Muris et al. [27]

\# No description of the pain as one or more of the following: burning, cutting, terrible, feeling of pressure, dull, boring

Table 5 Calculation of post-test probability of categories using category-oriented likelihood ratios in approaching nonacute abdominal pain

According to table 3, post-test probability of organic diseases category in case 2 after negative answer about 5 key discriminatory questions in history taking can be calculated as:

1. Pre-test odds $=\frac{0.14}{1-0.14}=0.16$

2. Post-test odds $=0.16 \times 0.78 \times 0.89 \times 0.77 \times 0.72 \times 0.89 \times 0.93=0.05$

3. Post-test probability $=\frac{0.05}{1+0.05}=4 \%$

Normal values of 3 key discriminatory clinical finding from table 4 again decrease the probability of organic category as:

1. Post-test odds $=0.05 \times 0.9 \times 0.92 \times 0.87=0.036$

2. Post-test probability $=\frac{0.036}{1+0.036}=3 \%$

By regarding pre-test probability of neoplasm sub category, its probability can be revised as:

1. Pre-test odds $=\frac{0.04}{1-0.04}=0.041$

2. $\quad$ Post-test odds $=0.041 \times 0.78 \times 0.89 \times 0.77 \times 0.72 \times 0.89 \times 0.93 \times 0.9 \times 0.92 \times 0.87=0.009$

3. Post-test probability $=\frac{0.009}{1+0.009}=0.8 \%$

purposes. Similar to the evidence-based algorithms and prediction rules used for revising the disease probability estimates, future evidence-based diagnostic algorithms can be developed to revise the probability estimate of the category of diseases in the light of experts' schemes. So, increasing diagnostic evidence can gradually fall in line with today's clinical education which advocates the teaching of approaches to clinical presentations rather than teaching diseases within separate disciplines. The efficacy of clinical education and clinical practice using these evidence-based algorithms can be evaluated in future.

The virtue of EBM is becoming increasingly recognized in the medical community especially among primary care physicians [30]. We hope that physicians will soon begin to apply easily accessible and evidence-based category-oriented LRs in their practice to make diagnoses more rapidly and accurately.

List of abbreviations used

EBM: Evidence-Based Medicine; LR: Likelihood Ratio: CBC: Complete Blood Count; ESR: Erythrocyte Sedimentation Rate; UTI: Urinary Tract Infection.

\section{Acknowledgements}

We express our appreciation to Dr. HamidReza Baradaran for his invaluable comments and edits in preparing the final revision of the manuscript.

\section{Author details}

${ }^{1}$ Evidence-Based Medicine \& Critical Thinking Working Team, Endocrinology and Metabolism Research Institute, Tehran University of Medical Sciences,

Tehran, IR Iran. ${ }^{2}$ Section of Neurosciences and Ethics, Chemical Injuries

Research Center, Baqiyatallah University of Medical Sciences, Tehran, IR Iran.

${ }^{3}$ Los Angeles Biomedical Research Institute at Harbor-UCLA Medical Center,

Torrance, CA, USA 


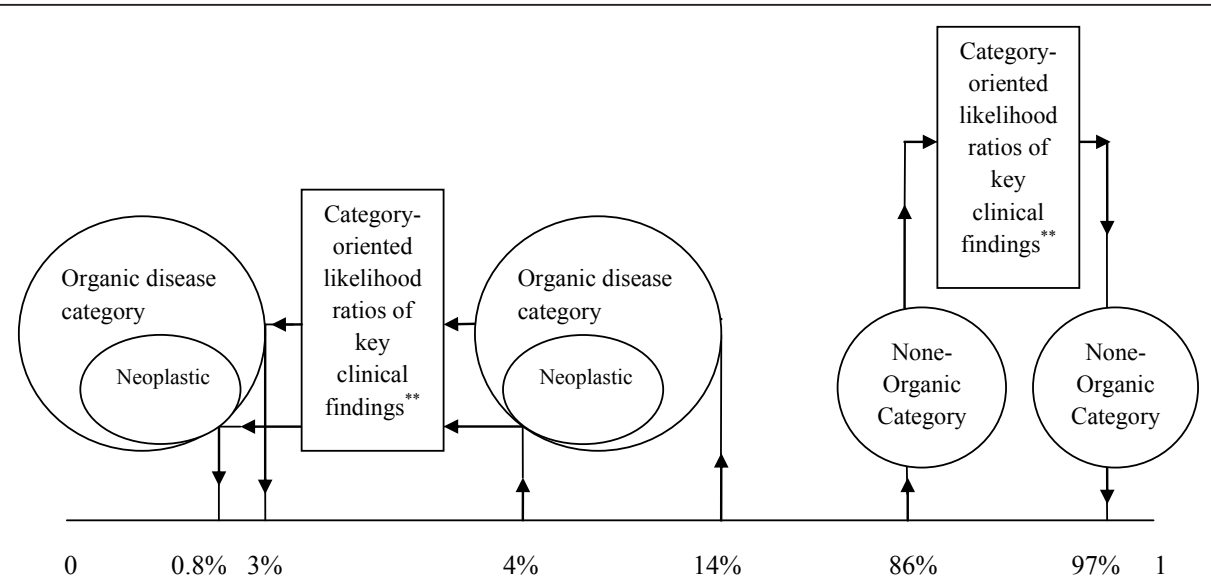

The of probability of the categories of diseases $\$$

Figure 2 Revising the probability estimates of organic and non-organic disease categories using category-oriented likelihood ratios in approach to patients complaining of non-acute abdominal pain*. * Category-oriented likelihood ratios of the key clinical findings and the probabilities calculated using relevant values derived from the result of a study by Muris et al. [27]. ** Key clinical findings in this example are: male gender, age $>60$ years, history of blood in stool, pain affecting sleep, no pain relief after defecation, no specific character of pain, weight loss $>1 \mathrm{~kg}$ in 4 weeks, white blood cell count $>10,000 \mathrm{~mm}-3$, erythrocyte sedimentation rate $>20 \mathrm{~mm}$ per hour, low hemoglobin level. ${ }^{\S}$ Arrows show the revising of the pre-test probability estimates to the post-test probability estimates.

\section{Authors' contributions}

HM had substantial contributions to concept and design, drafting the manuscript, revising it critically for important intellectual content and writing and final approval of the version to be published. MR had substantial contributions to concept and design, drafting the manuscript, revising it critically for important intellectual content and writing and final approval of the version to be published. MR had substantial contributions to concept and design, drafting the manuscript, revising it critically for important intellectual content and writing and final approval of the version to be published. AS developed the main concept and hypothesis, had substantial contributions to concept and design, drafting the manuscript, revising it critically for important intellectual content and writing and final approval of the manuscript.

\section{Authors' information}

- Hamideh Moosapour (M.D., Student of medical education) research assistant, EBM \& Critical Thinking Working Team, Endocrinology and Metabolism Research Center, Tehran University of Medical Sciences. - Mohsin Raza (M.D., PhD) the founder and current director of the faculty development program at the Baqiyatallah University of Medical Sciences and associate professor of neuroscience, at the Section of Neurosciences and Ethics, Chemical Injuries Research Center, Baqiyatallah University of Medical Sciences

- Mehdi Rambod (MD), post-doctoral fellow at Los Angeles Biomedical Research Institute at Harbor-UCLA Medical Center, Torrance, CA, USA - Akbar Soltani (M.D., M.A. in Philosophy, student of medical education) associate professor of endocrinology and the head of EBM \& Critical Thinking Working Team, Endocrinology and Metabolism Research Center, Tehran University of Medical Sciences

\section{Competing interests}

The authors declare that they have no competing interests.

Received: 21 December 2009 Accepted: 17 November 2011 Published: 17 November 2011

\section{References}

1. Alexander EK: Perspective: moving students beyond an organ-based approach when teaching medical interviewing and physical examination skills. Acad Med 2008, 83(10):906-909.
2. Hatala R, Smieja M, Kane SL, Cook DJ, Meade MO, Nishikawa J: An evidence-based approach to the clinical examination. J Gen Intern Med 1997, 12(3):182-187.

3. Fagan MJ, Griffith RA: An evidence-based physical diagnosis curriculum for third-year internal medicine clerks. Acad Med 2000, 75(5):528-529.

4. Wilson BE: Performance-based assessment of internal medicine interns: evaluation of baseline clinical and communication skills. Acad Med 2002, 77(11):1158.

5. Gruppen LD, Wolf FM, Billi JE: Information gathering and integration as sources of error in diagnostic decision making. Med Decis Making 1991, 11(4):233-239.

6. Friedman MH, Connell KJ, Olthoff AJ, Sinacore JM, Bordage G: Medical student errors in making a diagnosis. Acad Med 1998, 73(10 Suppl): S19-21.

7. Benbassat J, Baumal R, Heyman SN, Brezis M: Viewpoint: suggestions for a shift in teaching clinical skills to medical students: the reflective clinical examination. Acad Med 2005, 80(12):1121-1126.

8. Del Mar C, Doust J, Glasziou P: Clinical thinking: Evidence, communication and decision-making. BMJ Books; 2006.

9. Elstein AS, Schwartz A: Clinical problem solving and diagnostic decision making: selective review of the cognitive literature. BMJ 2002, 324(7339):729-732

10. Ahmadi-Abhari S, Soltani A, Hosseinpanah F: Knowledge and attitudes of trainee physicians regarding evidence-based medicine: a questionnaire survey in Tehran, Iran. Journal of evaluation in clinical practice 2008, 14(5):775-779.

11. Soltani A, Moayyeri A: What constitutes clinical evidence? A dynamic approach to clinical diagnosis. Canadian family physician Medecin de famille canadien 2 005, 51:1578-1579, 1582-1573.

12. Jaeschke R, Guyatt GH, Sackett DL: Users' guides to the medical literature. III. How to use an article about a diagnostic test. B. What are the results and will they help me in caring for my patients? The Evidence-Based Medicine Working Group. JAMA 1994, 271(9):703-707.

13. Bhandari M, Guyatt GH: How to appraise a diagnostic test. World I Surg 2005, 29(5):561-566

14. Gill CJ, Sabin L, Schmid CH: Why physicians are natural bayesians. BMJ 2005, 330(7499):1080-1083.

15. Goodman SN: Toward evidence-based medical statistics. 2: The Bayes factor. Ann Intern Med 1999, 130(12):1005-1013.

16. Deeks JJ, Altman DG: Diagnostic tests 4: likelihood ratios. BMJ 2004, 329(7458):168-169. 
17. Bent $S$, Nallamothu BK, Simel DL, Fihn SD, Saint S: Does this woman have an acute uncomplicated urinary tract infection? Jama 2002, 287(20):2701-2710.

18. Kempainen RR, Migeon MB, Wolf FM: Understanding our mistakes: a primer on errors in clinical reasoning. Med Teach 2003, 25(2):177-181.

19. Coderre S, Mandin H, Harasym PH, Fick GH: Diagnostic reasoning strategies and diagnostic success. Medical education 2003, 37(8):695-703.

20. Richardson WS, Wilson MC, Guyatt GH, Cook DJ, Nishikawa J: Users' guides to the medical literature: XV. How to use an article about disease probability for differential diagnosis. Evidence-Based Medicine Working Group. JAMA 1999, 281(13):1214-1219.

21. Fauci AS, Braunwald E, Kasper DL, Hauser SL, Longo DL, Jameson JL, Loscalzo J: Harrison's Principles of Internal Medicine McGraw-Hill Medical; 2008.

22. Mandin H, Harasym P, Eagle C, Watanabe M: Developing a "clinical presentation" curriculum at the University of Calgary. Acad Med 1995, 70(3):186-193.

23. Kostopoulou O, Oudhoff J, Nath R, Delaney BC, Munro CW, Harries C, Holder R: Predictors of diagnostic accuracy and safe management in difficult diagnostic problems in family medicine. Med Decis Making 2008, 28(5):668-680

24. Croskerry P: The importance of cognitive errors in diagnosis and strategies to minimize them. Acad Med 2003, 78(8):775-780.

25. Wagner JM, McKinney WP, Carpenter JL: Does this patient have appendicitis? JAMA 1996, 276(19):1589-1594.

26. Soltani A, Moayyeri A: Towards evidence-based diagnosis in developing countries: the use of likelihood ratios for robust quick diagnosis. Annals of Saudi medicine 2006, 26(3):211-215.

27. Muris JW, Starmans R, Fijten GH, Crebolder HF, Schouten HJ, Knottnerus JA: Non-acute abdominal complaints in general practice: diagnostic value of signs and symptoms. Br J Gen Pract 1995, 45(395):313-316.

28. Sackett $\mathrm{DL}$ : The rational clinical examination. A primer on the precision and accuracy of the clinical examination. Jama 1992, 267(19):2638-2644.

29. Simel LDavid, Rennie Drummond, Keitz ASheri: The Rational Clinical Examination:Evidence-Based Clinical Diagnosis McGraw-Hill Medical; 2008.

30. Del Mar C, Glasziou P: How many conditions can a GP screen for? BMJ 2003, 327(7424):1117.

\section{Pre-publication history}

The pre-publication history for this paper can be accessed here:

http://www.biomedcentral.com/1472-6920/11/94/prepub

doi:10.1186/1472-6920-11-94

Cite this article as: Moosapour et al:: Conceptualization of category-

oriented likelihood ratio: a useful tool for clinical diagnostic reasoning.

BMC Medical Education 2011 11:94.

\section{Submit your next manuscript to BioMed Central and take full advantage of:}

- Convenient online submission

- Thorough peer review

- No space constraints or color figure charges

- Immediate publication on acceptance

- Inclusion in PubMed, CAS, Scopus and Google Scholar

- Research which is freely available for redistribution

Submit your manuscript at www.biomedcentral.com/submit 